\title{
Emission of Visible Light by Hot Dense Metals
}

\author{
Richard M. MORE ${ }^{1,2)}$, Motoshi GOTO ${ }^{3)}$, Frank GRAZIANI ${ }^{2)}$, Pavel NI ${ }^{1)}$ and Hitoki YONEDA ${ }^{4)}$ \\ 1) Lawrence Berkeley National Laboratory, Berkeley, California 94720, USA \\ ${ }^{2)}$ Lawrence Livermore National Laboratory, Livermore, California 94550, USA \\ ${ }^{3)}$ National Institute for Fusion Science, Toki, Gifu 509-5292, Japan \\ 4) University of Electro-Communications, Chofu, Tokyo 182-8585, Japan
}

(Received 9 December 2009 / Accepted 2 March 2010)

\begin{abstract}
We consider the emission of visible light by hot metal surfaces having uniform and non-uniform temperature distributions and by small droplets of liquid metal. The calculations employ a nonlocal transport theory for light emission, using the Kubo formula to relate microscopic current fluctuations to the dielectric function of the material. We describe a related algorithm for calculating radiation emission in particle simulation of hot fusion plasmas.
\end{abstract}

(C) 2010 The Japan Society of Plasma Science and Nuclear Fusion Research

Keywords: incandescent emission of light, polarized emission, pyrometry, hot metal, warm dense matter

DOI: $10.1585 /$ pfr.5.S2007

\section{Introduction}

You can see light from glowing hot metals - the kitchen stove, tungsten filaments, or the walls of fusion experiments. It's a disappointment not to find a satisfactory theory for this emission by hot metals in our favorite books of electro-dynamics [1], optics [2] and solid-state physics [3], or even in general textbooks of physics [4].

A metal is a dense plasma having a plasma frequency $\hbar \omega_{\mathrm{p}} \sim 10-15 \mathrm{eV}$. Visible light has $\hbar \omega \sim 2 \mathrm{eV}$, so $\hbar \omega \ll \hbar \omega_{\mathrm{p}}$. A plasma is overdense to light of frequency $\omega$ if $\omega<\omega_{\mathrm{p}}$ and $\operatorname{Re}[\varepsilon(\omega)]<0$, where $\varepsilon(\omega)$ is the frequency-dependent dielectric function. Light reflects from a smooth metal surface and only an evanescent wave penetrates over a skin depth $\delta \sim c / \omega_{\mathrm{p}} \sim 10 \mathrm{~nm}$. Electrons deep in the metal emit much less light than electrons near the surface even if they have the same local density-temperature conditions; emission is suppressed because the electrons only connect to the exponential tail $\sim \exp (-x / \delta)$ of the light waves.

We consider metals such as W, Au, Al, Pd, Mo, or Pt. The cold-matter optical properties are summarized by a dielectric function $\varepsilon(\omega)$ [5]. When they are heated to "Warm Dense Matter" (WDM) temperatures $\sim 1 \mathrm{eV}$, we will have to guess $\varepsilon(\omega)$ and compare to experiments. In order to do that comparison we need equations that apply for an arbitrary dielectric function $\varepsilon(\omega)$. At high temperatures metals evaporate and expand. For very hot metal, the expansion is a release or rarefaction flow; hydrodynamic calculations predict the $\rho, T$ profile [6]. The metal cools as it expands, so the outer layers are cooler than the interior.

In WDM experiments, the temperature has strong gradients. WDM density and temperature may vary over distances much less than the wavelength of light. In this case the emission cannot be calculated from absorption by Kirchhoff's law, so we need a microscopic emission theory.

The equation of radiative transfer gives the radiation energy flow as an integral along light ray paths [6]. This equation applies when the spatial gradients are small over a photon mean free path and very small over the wavelength of light. Neither condition is satisfied for most WDM experiments. In addition, light rays are undefined in overdense plasma because the index of refraction $\sqrt{\varepsilon}=n+\mathrm{i} k$ is a complex number. Radiative transfer is a form of heat conduction, described by Fourier's law, $q=-k \operatorname{grad} T$, and obeys certain general rules: for example, when heat flows from $T_{\text {Hot }}$ to $T_{\text {Cold }}$, then between those points the temperature is necessarily between $T_{\text {Hot }}$ and $T_{\text {Cold }}$.

Nonlocal transport is a different type of heat flow, in which a conduction channel (usually having a limited channel capacity or flux limit) carries heat energy limited by an injection impedance at the hot end and an extraction impedance at the cool end. The channel might traverse colder or hotter matter without being coupled enough to equilibrate locally. In the cases we are considering, the conduction channel is the overdense electromagnetic field. In this nonlocal transport theory the emitted radiation is given by an integral over the sources, which are fluctuating microscopic currents. The theory naturally invokes the Weiner-Kinchin theorem for stationary random processes and the Kubo formula (fluctuation-dissipation theorem). With these equations the emission is given by an integral over the local dielectric function.

In this paper we discuss emission of light in three situations of progressively increasing temperature: small droplets of liquid metal, emission from hot metal surfaces 
and finally, emission from hot fusion plasmas described by Molecular Dynamics (MD) particle simulation.

\section{Emission by Small Droplets of Liq- uid Metal}

Accelerator-heated WDM experiments on an experimental facility in the Lawrence Berkeley National Laboratory rapidly heat a submicron-thick foil to several thousand K. The foil appears to break into small droplets of liquid metal and time-resolved emission spectra are measured. We would like to extract information from those spectra to decide the temperature of the hot material and, if possible, the size of the droplets. At the conditions of the experiments the metal inside the droplets probably has uniform density and temperature.

In this case Kirchhoff's law applies and we can calculate the emission from the absorption cross-section $A(\omega)$. The total power emitted into $4 \pi$ steradians is

$$
\frac{\mathrm{d} E}{\mathrm{~d} t}=\int \frac{(\hbar \omega)^{3}}{\pi^{2} \hbar^{3} c^{2}} \frac{A(\hbar \omega)}{\left(\mathrm{e}^{\hbar \omega / k T}-1\right)} \mathrm{d} \hbar \omega .
$$

$A(\hbar \omega)$ is the absorption cross-section for a plane wave approaching the droplet. We calculate $A(\hbar \omega)$ from Mie theory of light scattering by a small droplet $[1,2,7]$.

The electromagnetic fields are represented as a series of vector spherical harmonic functions. Inside the droplet there is an evanescent wave, which has a complex wavevector

$$
K=(\omega / c) \sqrt{\varepsilon}
$$

To evaluate the formulas we need Bessel functions $j_{\ell}(K r)$ for complex wave-vector $K$ and for large index $\ell$ (up to 100). To generate these functions we use a recursion formula iterating down $(\ell \rightarrow \ell-1)$ for the Bessel function $j_{\ell}$ and iterating up $(\ell \rightarrow \ell+1)$ for the Neumann function $n_{\ell}$. A Bessel-function theorem helps evaluate the volumeintegral of the Joule heating to calculate the absorption. We evaluate the formulas using measured dielectric functions [5] which are valid for room-temperature material.

A useful check of the computer code is the comparison to an analytic formula (Eq. 93-4 of reference [4]) valid for droplets that are small compared to the wavelength; our code agrees accurately with this formula for $R \ll \lambda$.

A comparison to geometrical optics agrees to a few percent for large spheres. Many textbooks warn us that geometrical optics is inaccurate for scattering of light from smooth spheres, due to interference phenomena associated with wave optics $[2,7,8]$. For large spheres, absorption or emission occurs mainly on the forward surface, so better agreement is not surprising.

Theory predicts anisotropic absorption from a planepolarized plane wave: absorption on the two sides where $\boldsymbol{E}$ points toward the sphere is stronger than absorption on the sides where $\boldsymbol{E}$ is parallel to the surface. This is because ppolarized light is absorbed better than s-polarized light (see below). The emission from a small sphere is isotropic and unpolarized. It is typically about $10 \%$ of the black-body emission.

The droplet calculations are only relevant in a limited temperature range between melting and boiling temperatures. At higher temperatures, evaporation or rarefaction flow will rapidly produce a nonuniform densitytemperature profile and we must calculate the emission without using detailed balance.

\section{Emission by a Uniform Hot Metal}

For a hot metal layer in which the temperature is uniform we can again calculate the emission from the absorption coefficient using Kirchhoff's law. This special case gives a useful test for the general theory of emission.

To calculate the absorption during reflection from a flat metal surface, one solves the Maxwell eqs. for the classical $\boldsymbol{E}, \boldsymbol{B}$ fields. The energy flow toward the material is $S_{x}$ $=x$-component of the Poynting vector, where $x$ is the direction into the material (the surface is the $y z$ plane). The energy absorption is

$$
\frac{\mathrm{d} S_{x}}{\mathrm{~d} x}=-\operatorname{Re}[\sigma(\omega)]\left\langle|\bar{E}(x)|^{2}\right\rangle
$$

$=$ local Joule heating. To evaluate this one needs the highfrequency conductivity $\sigma(\omega)$, related to the dielectric function by

$$
\varepsilon(\omega)=1+4 \pi \mathrm{i} \sigma(\omega) / \omega
$$

so

$$
\operatorname{Re}[\sigma(\omega)]=\omega / 4 \pi \operatorname{Im}[\varepsilon(\omega)] .
$$

Theory predicts that p-polarized absorption is larger than s-polarized absorption

$$
A_{\mathrm{p}}>A_{\mathrm{s}} \text {. }
$$

The s- and p-polarized absorptions differ, in part, because p-polarized light induces a surface-charge density.

Laser experiments readily measure absorption $A_{\mathrm{s}}, A_{\mathrm{p}}$ and even the rotation of the polarization ellipse if the incident light is mixed s- and p-polarized. This technique of ultra-short pulse pump-probe ellipsometry can give quantitative information about laser-heated materials with subpicosecond time resolution [9]. The absorption theory is well-tested by laser experiments and measurements on cold matter.

If the metal has constant temperature and density, the absorption coefficients are given by the Fresnel formulas, evaluated with the optical constants $n$ and $k$ determined by $\varepsilon(\omega)=(n+\mathrm{i} k)^{2}$. However the Fresnel formulas fail as soon as the metal surface begins to expand [10].

\section{Emission by a Nonuniform Metal Surface}

A general theory of emission is more difficult. We examine the absorption and emission for a normal mode 
of the radiation field characterized by a free-space wavevector $\left(k_{x}, k_{y}, k_{z}\right)$. Light absorption is coherent and stimulated emission is a coherent reduction of the absorption, but for the materials considered here the spontaneous emission is incoherent.

Electric and magnetic fields made by the sources are linearly related to the sources,

$$
\boldsymbol{E}=\boldsymbol{G}_{E} \circ \boldsymbol{j} \quad \text { and } \quad \boldsymbol{B}=\boldsymbol{G}_{B} \circ \boldsymbol{j},
$$

where the Green's functions $\boldsymbol{G}_{E}$ and $\boldsymbol{G}_{B}$ depend on polarization (selected by the direction of the current fluctuations $j$ ). The Green's functions include the coherent response of the medium (overdense "refraction", absorption, stimulated emission) as decribed by the dielectric function $\varepsilon(\omega)$. The G's are constructed as products of matrices for layers of target material; they decrease like $\exp (-\operatorname{Re}[\gamma] x)$. Here $\gamma$ is the $x$-component of the wave-vector inside the metal,

$$
\gamma^{2}=\left(\omega^{2} / c^{2}\right)\left[\sin ^{2} \theta-\varepsilon(\omega)\right]
$$

Of course the electric field oscillates rapidly and its time-average is zero. We examine a quadratic quantity, the Poynting vector

$$
\boldsymbol{S}=c(\boldsymbol{E} \times \boldsymbol{B}) / 4 \pi .
$$

$S$ is a sum over the incoherent sources, which are microscopic currents in the material. The emission can be written symbolically:

$$
\boldsymbol{S} \sim(c / 4 \pi) \int \boldsymbol{G}_{E} \times \boldsymbol{G}_{B} \circ<\boldsymbol{j} \circ \boldsymbol{j}>\mathrm{d} x
$$

Here $\langle\boldsymbol{j} \circ \boldsymbol{j}\rangle$ is the current fluctuation, related to the current-current autocorrelation function by the WeinerKinchin theorem. The Kubo relation connects this quantity to the real part of the AC electrical conductivity $\operatorname{Re}[\sigma(\omega)]$ which is in turn proportional to $\operatorname{Im}[\varepsilon(\omega)]$, the imaginary part of the dielectric function.

The difficult part of this calculation is the calculation of the Green's functions, especially for p-polarized light, but there is a simple and convincing way to check the calculation.

For a uniform metal plate (having constant composition, density and temperature) the emitted spectrum exactly equals the absorption from a blackbody spectrum having the same temperature, i.e., the power emitted from a given layer into a given solid angle in a given frequency range equals the power absorbed in the same layer from the same solid angle at the same frequencies.

The equations predict a phenomenon of overdense radiation transport which we have not seen discussed in the scientific literature. When a source at depth $x_{S}$ makes electric and magnetic fields as in Eq. (5), some of the energy of these fields is absorbed in a second layer at depth $x_{T}$. The Green's functions describe that absorption through the $\operatorname{Im}[\varepsilon]$ in the wave-equation. However, if the material temperature is uniform (homogeneous), the second region at
$x_{T}$ also emits radiation and some of that penetrates back to $x_{S}$ and is absorbed there. These two absorptions are exactly equal if the temperatures $T\left(x_{S}\right)$ and $T\left(x_{T}\right)$ are equal, and that is a powerful consistency check for the theory (Onsager symmetry). If the two temperatures are not equal, there is a net heat conduction from hot to cold. Because the overdense radiation field typically does not have a large energy-density (compared to the material energy-density) this overdense transport cannot be a strong correction to the normal electron heat conduction, but it is a powerful check of our calculations to find that it obeys the principle of detailed balance.

The calculations described here predict that emission from hot metals (having smooth and clean surfaces) should be strongly p-polarized, when seen from a sufficient angle from the normal.

\section{Polarization and Pyrometry}

Polarized emission from metals was observed many years ago (Millikan [11]), but apparently is not well known: it is not even mentioned in modern textbooks [2]. Probably magnetic fusion machines are filled with polarized visible light emitted by hot metal on the limiter, divertor, etc.

In the National Institute for Fusion Science, M. Goto et al. recently measured polarized emission for electronbeam heated foils of $\mathrm{W}$ metal. The experiments observed polarization resolved spectra of radiation from the tungsten surface which was heated by an electron beam. The viewing angle to the surface was 75 degree from normal to the surface. One component is polarized parallel (\|) to the surface and the other $(\perp)$ is perpendicular to it. The temperature was estimated from the spectra. The radiation is strongly polarized. The experiments study the polarization at different temperatures and some temperaturedependence of the polarization was observed at the highest temperatures. In the Lawrence Berkeley National Laboratory and the University of Electro-Communication, $\mathrm{H}$. Yoneda and P. Ni have observed polarized emission from electrically heated strips of several metals.

Optical pyrometry is a well-known way to determine temperature from emitted light. The optical pyrometer has limited time-response and temperature sensitivity because it must collect enough photons to obtain accurate data. The usual optical pyrometer is an imperfect thermometer for another reason: one measures the emitted intensity $I\left(\omega_{j}\right)$ at $N$ wavelengths, but there are almost always $N+1$ unknowns: the $N$ emissivities $E\left(\omega_{j}\right)$ and the surface temperature $T$. One can only extract a temperature by making some assumption about the frequency-dependence of the surface emissivity $E(\omega)$.

For this reason we propose a polarization pyrometer, which will measure emission of the two polarizations at several angles. In this way one can collect enough data to unfold the unknown dielectric function and the unknown 
temperature. This is especially important for WDM where we do not a priori know the emissivity $E(\omega)$ or dielectric function $\varepsilon(\omega)$.

\section{Radiation in Particle Simulations}

Another question about emission theory arises in molecular dynamics (MD) particle simulation which we use to study hot plasmas at fusion ignition conditions. This work uses large computers to perform simulations with $10^{5}-10^{6}$ electrons and ions $[12,13]$. Previous particle simulations have been performed to study charged-particle paircorrelations, especially for strongly-coupled plasmas, but we want to extend the method to study atomic-scale phenomena that occur in fusion plasmas. We plan to include fusion reactions and the slowing of fusion products, emission and absorption of radiation and other atomic processes involving bound electrons on high- $Z$ impurities. The particle simulations are based on classical MD of electrons and ions, but the close collisions are quantum mechanical. For example, fusion reactions only occur because of quantum tunneling, and the radiation processes also involve quantum effects.

The calculation of radiation poses a simple but important question: how to modify a classical simulation so as to obtain the (quantum) black-body spectrum when the simulation reaches equilibrium. (A purely classical simulation would necessarily give the incorrect Rayleigh-Jeans spectrum). Here we briefly mention two complementary approaches to the description of radiative processes.

\subsection{Close collisions: hybrid MD/MC algo- rithm}

The quantum effects are very important for highenergy photons (having $\hbar \omega>k T$ ) which are most important energetically. They are emitted or absorbed in "close" collisions with small impact parameters. To describe emission and absorption of high-energy photons, we use a hybrid Molecular Dynamics/Monte Carlo method. Quantum or semiclassical cross-sections are used to describe the radiative processes which occur during a close collision. When the classical MD brings an electron to within a small distance $R_{B}$ of an ion, control is passed to a subroutine (SB $=$ "small ball") which forms a conditional probability $P_{\text {cond }}$ to emit or absorb a photon.

SB Algorithm \# 1

$$
P_{\text {cond }}=\frac{\sigma_{K}}{\pi R_{B}^{2}}
$$

SB Algorithm \# 2 ( $\ell$-dependent cross-section)

$$
P_{\text {cond }}=\frac{\sigma_{1}}{\pi(2 \ell+1) \hbar^{2} / m^{2} v^{2}}
$$

The first algorithm is used with the Kramers cross-section $\sigma_{K}$. MC tests are used to decide between absorption and emission and to decide the energy $\hbar \omega$ of the photon, guided by conditional probabilities obtained from the cross-section [12]. The second algorithm requires a more detailed cross-section that resolves the emission or absorption by the angular momentum of the incoming electron. The angular momentum $\ell$ is determined by the classical position and velocity when the electron reaches the radius $r=R_{B}$.

\subsection{Soft collisions: coupling to classical nor- mal modes}

Emission of low-energy photons (visible and ultraviolet light) can be handled in a different way. Since $\hbar \omega \leq k T$ for this case, a classical calculation may suffice.

We start from the normal-mode representation of the electromagnetic field, as in non-relativistic (Coulombgauge) quantum electrodynamics (QED). The electromagnetic field is obtained from a vector potential which is expanded in field amplitudes $a_{k \lambda}$ (in QED $a_{k \lambda}$ is a photon annihilation operator).

$$
A(\vec{r}, t)=\sum_{k, \lambda} \hat{e}_{k, \lambda} C_{k}\left[a_{k, \lambda} \mathrm{e}^{\mathrm{i} \vec{k} \cdot \vec{r}}+a_{k, \lambda}^{+} \mathrm{e}^{-\mathrm{i} \vec{k} \cdot \vec{r}}\right]
$$

The equation of motion for the field amplitudes is:

$$
i \hbar \frac{\partial a_{k, \lambda}}{\partial t}=\hbar \omega a_{k, \lambda}-\sum_{p} \frac{q}{c}\left(v_{p} \cdot e_{k, \lambda}\right) C_{k} \mathrm{e}^{-\mathrm{i} k \cdot r_{\mathrm{p}}(t)}
$$

Here the subscript $\mathrm{p}$ denotes one particle (usually an electron), $r_{\mathrm{p}}(\mathrm{t})$ and $v_{\mathrm{p}}(\mathrm{t})$ are the particle position and velocity vectors, $\lambda=$ photon polarization, and

$$
C_{k}=\left(\frac{2 \pi \hbar c^{2}}{V \omega}\right)^{1 / 2}
$$

where $V=$ quantization volume. Eq. (12) omits a secondorder term that generates Compton scattering. The change of energy for the normal mode specified by $k$ and $\lambda$ is

$$
\begin{aligned}
\hbar \omega \frac{\partial n_{k, \lambda}}{\partial t}= & -\sum_{p} q\left(v \cdot e_{k, \lambda}\right)\left(\frac{\mathrm{i} \omega}{c}\right) \\
& \times\left[a_{k \lambda}^{+} \mathrm{e}^{-\mathrm{i} \vec{k} \cdot \vec{r}_{\mathrm{p}}(t)}-a_{k \lambda} \mathrm{e}^{\mathrm{i} \vec{k} \cdot \overrightarrow{\mathrm{p}}_{\mathrm{p}}(t)}\right]
\end{aligned}
$$

We could solve Eq. (12) numerically, but that would require a small time-step. Instead we Fourier analyze $a_{k \lambda}(\mathrm{t})$ and project out the emission at frequencies near $\omega=c k$. (Lower frequencies $\sim k \cdot v$ correspond to modifications of the near-field Coulomb potential, e.g., magnetic effects $v / c$ ). We then multiply by the photon density of states (and sum over polarizations), i.e. we form

$$
\mathrm{d} P=\sum_{\lambda} \frac{4 \pi k^{2} \mathrm{~d} k}{8 \pi} \hbar \omega\left[\frac{\partial n_{k, \lambda}}{\partial t}\right]_{\text {emission }}
$$

and obtain

$$
\frac{\mathrm{d} P(\omega)}{\mathrm{d} \omega}=\sum_{p} \frac{2}{3} \frac{q^{2}}{c^{3}} \frac{\omega^{2}}{\pi} \int_{0}^{\infty}\left\langle v_{p}(0) \cdot v_{p}(\tau)\right\rangle \mathrm{e}^{\mathrm{i} \omega \tau} \mathrm{d} \tau
$$


This is also a current-current correlation function, closely related to the Kubo formula for the electrical conductivity of the plasma.

The current-current correlation function supplied by the particle MD calculation will include various correlation effects produced by Coulomb forces in the plasma. In this case we again find the emission is expressed in terms of the AC conductivity without the complications of overdense material and nonlocal transport.

\section{Summary and Conclusions}

The main points of this research are

1) We study the general theory of emission of radiation by hot dense matter.

2) Our computer codes predict light emission using material descriptions obtained from hydrodynamic or particle MD codes.

3) They predict that light emitted from a smooth clean hot metal surface is predominantly p-polarized.

4) The codes provide a tool for improving the interpretation of pyrometry measurements of emission and finding a more convincing determination of WDM plasma temperatures from pyrometry measurements.

\section{Acknowledgments}

This work was partially supported by the Office of $\mathrm{Fu}$ sion Energy Sciences, of the U.S. Department of Energy under Contract No. DE-AC02-05CH11231 and partially supported by the Lawrence Livermore National Laboratory under contract DE-AC52-07NA27344 through the LDRD Program at LLNL under project code 09-SI-011.
[1] J. D. Jackson, Classical Electrodynamics, 3rd Ed. (J. Wiley, Hoboken, NJ 1999).

[2] M. Born and E. Wolf, Principles of Optics, 5th Ed. (Pergamon Press, Oxford 1975).

[3] C. Kittel, Introduction to Solid-State Physics, 3rd Ed. (Wiley, NY 1967); F. Seitz, Modern Theory of Solids (Dover, NY 1987); W. Jones and N. H. March, Theoretical Solid State Physics (Dover, NY 1985).

[4] E. Landau and I. M. Lifshitz, Electrodynamics of Continuous Media, 2nd Ed. (Pergamon Press, Oxford 1984). See section 93, eq. 93-4

[5] E. Palik, Handbook of Optical Constants of Solids (Academic Press 1985).

[6] Ya. Zel'dovich and Yu. Raizer, Physics of Shock Waves and High-Temperature Hydrodynamic Phenomena (Dover 2002).

[7] H. C. Van de Hulst, Light Scattering by Small Particles (Dover Publications, New York 1981).

[8] A. Sommerfeld, Optics (Academic Press, NY 1954).

[9] H. Yoneda, H. Morikami, K. Ueda and R. More, Phys. Rev. Lett. 91, 075004 (2003); H. Yoneda, H. Morikomi, K.-I. Ueda and R. More, J. Plasma Fusion Res. 79, 449 (2003).

[10] H. Morikami, H. Yoneda, K.-I. Ueda and R. M. More, Phys. Rev. E70, 035401 (2004).

[11] R. A. Millikan, Phys. Rev. 3, 81 (1895); Phys. Rev. 3, 177 (1895); see also A. G. Worthing, J. Opt. Soc. Am. \& RSI 13, 635 (1926).

[12] R. More, F. Graziani, J. Glosli and M. Surh, High Energy Density Phys. 6, 29 (2010); J. Glosli, F. Graziani, R. More, M. Murillo, F. Streitz and M. Surh, J. Phys. A42, 214030 (2009).

[13] R. More, F. Graziani, J. Glosli and M. Surh, High Energy Density Phys. 6, 29 (2010). 